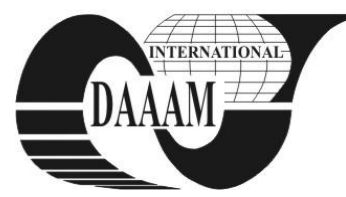

Annals of DAAAM for 2011 \& Proceedings of the 22nd International DAAAM Symposium, Volume 22, No. 1, ISSN 1726-9679 ISBN 978-3-901509-83-4, Editor B. Katalinic, Published by DAAAM International, Vienna, Austria, EU, 2011 Make Harmony between Technology and Nature, and Your Mind will Fly Free as a Bird Annals \& Proceedings of DAAAM International 2011

\title{
CYLINDRICAL PACKAGES SIMULATIONS
}

\section{PRACEK, S[tanislav]; SLUGA, F[ranci]; MOZINA, K[lemen] \& FRANKEN, G[regor]}

\begin{abstract}
Stability of the yarn unwinding directly affects the efficiency of the textile production process and the quality of the final product. A package with an optimal shape will result in an optimal shape of the balloon. In addition, the yarn tension will be small and steady even at high unwinding velocity.

Key words: cylindrical packages, winding angle, yarn unwinding, angular velocity, unwinding speed
\end{abstract}

\section{INTRODUCTION}

During the yarn unwinding from a stationary package, the yarn slides on the surface of the package before it lifts off to form a balloon. The point where the yarn begins to slide is known as the unwinding point, while the point where the yarn lifts off from the surface is known as the lift-off point. On this section of the yarn, i.e. between the unwinding point and the lift-off point, the tension in the yarn drops from its value in the balloon (at the lift-off point) to its residual value, defined as the tension of the yarn inside the package.

\section{THEORETICAL PART}

The problem of yarn motion on the package surface during the unwinding can be treated in analogy with the motion of the yarn forming the balloon between the lift-off point and the eyelet, through which the yarn is being pulled.

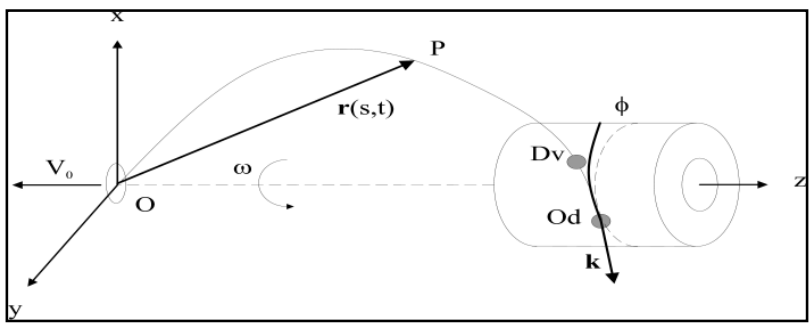

Fig.1. Mechanical setup in overend yarn unvinding from cilimdrical package

The yarn is being withdrawn with velocity $\mathrm{V}$ through an eyelet, where we also fix the origin $\mathrm{O}$ of our coordinate system (Fig.1). The yarn is rotating aroun the $\mathrm{z}$ axis with an angular velocity $\omega$. At the lift-off point Dv the yarn lifts from the package and forms a balloon. At the unwinding point Od the yarn starts to slide on the surface of the package. Angle $\phi$ is the winding angle of the yarn on the package.

The general equation of motion for the yarn was derived and justified in one of the previous works (Padfield, 1958; Barr \& Catling, 1976; Kothari \& Leaf, 1979 ; Fraser \& Ghosh \& Batra, 1992):

$$
\rho\left(D^{2} r+2 \omega \times D r+\omega \times(\omega \times r)+\dot{\omega} \times r\right)=\frac{\partial}{\partial s}\left(T \frac{\partial r}{\partial s}\right)+f
$$

The position vector $\mathbf{r}$ points from the origin of the coordinate system to a chosen point along the yarn, $\rho$ is the linear density of the yarn mass, $\omega$ is the angular velocity vector of the spinning coordinate system in which the yarn is being described and which points along the z-axis, D is the operator of the total time derivative which follows the motion of the point inside the spinning coordinate system, $\mathrm{D}=\partial / \partial \mathrm{t}_{\mathrm{r}, \mathrm{z}, \mathrm{z}}-\mathrm{V} \partial / \partial \mathrm{s}$, $\mathrm{T}$ is the mechanical tension, $\mathbf{f}$ is the linear density of external forces. In the part of the yarn which forms the balloon, $\mathbf{f}$ is the air drag force:

$$
f=-\frac{1}{2} c_{u} \rho d\left|v_{n}\right| v_{n},
$$

where $c_{u}$ is the effective air-drag coefficient, $d$ is the yarn diameter, $\mathbf{v}_{\mathrm{n}}=\mathbf{v}-(\mathbf{v} \cdot \mathbf{t}) \mathbf{t}$ is the normal component of the yarn velocity ( $t$ is the unit tangent vector to the yarn at the given point).

When, We study the unwinding of yarn from a cylindrical package (Figure 1). The yarn in the layer which is being unwound has a winding angle $\phi$. We will show that the winding angle determines the angular velocity $\omega$, if the unwinding velocity $\mathrm{V}$ and the package radius $\mathrm{c}$ are known. The derivation is applicable in the quasi-stationary approximation which consists of neglecting the variation of system parameters (in particular of the winding angle $\phi$ ) during a single period of the balloon motion around the $\mathrm{z}$ axis.

In cylindrical packages the relation between the angular velocity of the yarn during unwinding $\omega$, the unwinding speed $\mathrm{V}$ and the package radius $\mathrm{c}$ at the lift-off point, where the yarn lifts off from the package surface, is (Praček, 2002):

$$
\omega=\frac{2 \pi}{t}=\frac{2 \pi V}{L-M}=\frac{V}{\frac{c}{\cos \phi}-c \tan \phi}=\frac{V \cos \phi}{c(1-\sin \phi)}
$$

\section{PRACTICAL PART}

We now present the results of the simulations. We investigated the effects of different unwinding velocities $\mathrm{V}$, package radii $\mathrm{c}$, and the winding angles $\phi$.

In Fig. 2 we show the tension during the unwinding at $\mathrm{V}=$ $1500 \mathrm{~m} / \mathrm{min}$ and at radius $\mathrm{c}=100 \mathrm{~mm}$ for different winding angles. For densely parallely wound packages $(\phi \sim 0)$ the tension is constant, as expected, since in parallely wound packages the angular velocity is approximately constant. In cross wound packages the velocity is time dependant. When the yarn unwinds backwards the angular velocity is higher as when the yarn unwinds forwards, which follows from Eq. (3). Since the tension strongly depends on the angular velocity, we obtain oscillations in the yarn tension. The abrupt change in the tension occurs when the lift-off points reaches an edge of the package. Such changes strain the yarn and can lead to damage. In the most extreme case, the yarn can break. In designing new 
package types it is therefore necessary to limit both the maximum tension in the yarn and the amplitude of oscillations during unwinding.

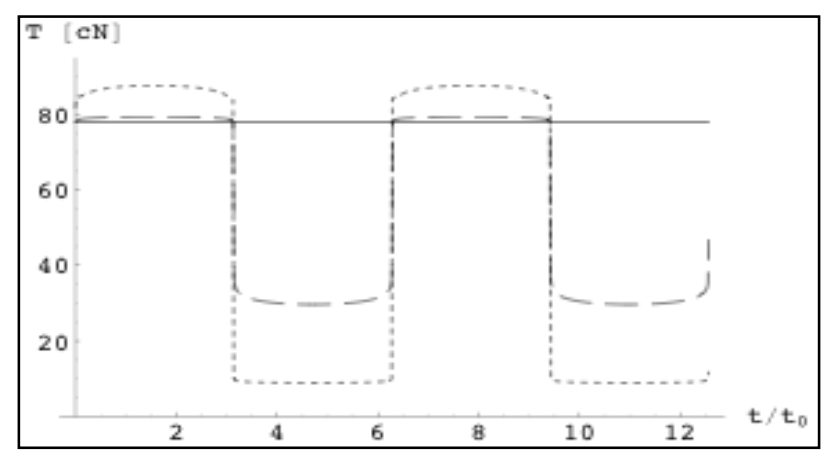

Fig. 2. Tension in the yarn during unwinding at $V=1500$ $\mathrm{m} / \mathrm{min}$ and at radius $c=100 \mathrm{~mm}$ for different winding angles: $\phi=0^{\circ}$ (solid line), $\phi=10^{\circ}$ (dashed line) and $\phi=20^{\circ}$ (dotted line)

In Fig. 3 we show unwinding from a similar package, but at higher unwinding velocity $V=2000 \mathrm{~m} / \mathrm{min}$. Tensions are in this case very high. Unwinding from such packages might not be possible.

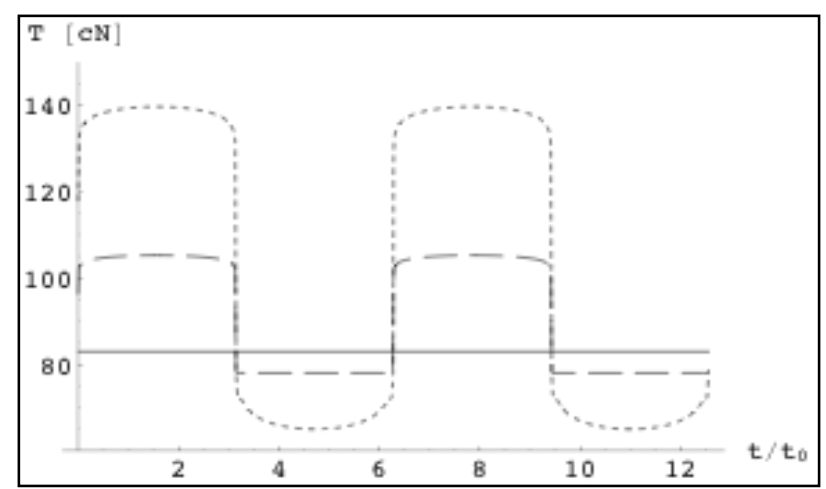

Fig. 3. Tension in the yarn during unwinding at $V=2000$ $\mathrm{m} / \mathrm{min}$ and at radius $c=100 \mathrm{~mm}$ for different winding angles: $\phi=0^{\circ}$ (solid line), $\phi=10^{\circ}$ (dashed line) and $\phi=20^{\circ}$ (dotted line)

In Fig. 4 we show a 3D plot of tension as a function of time and package radius. For large radius packages the tension is significantly reduced. Therefore it is still possible to unwind at very high unwinding velocities from packages with sufficiently large smallest radius (as determined by the radius of the tube on which the yarn is wound). We have determined that the minimum radius should be around $150 \mathrm{~mm}$ in order to keep the tension below $50 \mathrm{cN}$.

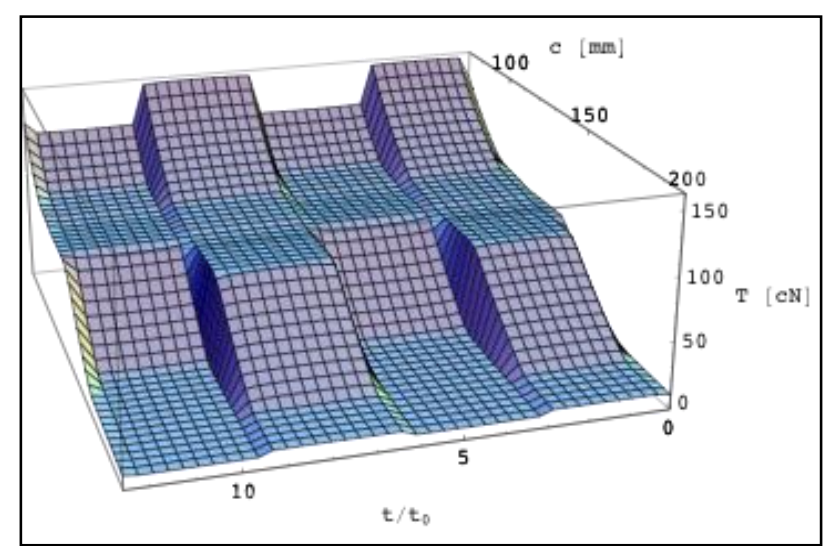

Fig. 4. Tension in the yarn as a function of package radius. $\mathrm{V}=2000 \mathrm{~m} / \mathrm{min}, \phi=5^{\circ}$
This limiting value of $50 \mathrm{cN}$ is chosen so that the largest deformation in the yarn is in the elastic regime, i.e. the tension in the yarn should be below $10 \%$ of the breaking tension. The values were determined experimentally. During unwinding tests we achieved up to $17 \%$ of the breaking tension without affecting the mechanical properties of the yarn: the breaking tension of the yarn after unwinding was not reduced. We determined the breaking tension to be $4.5 \mathrm{~N}=450 \mathrm{cN}$, from which follows the estimate of $50 \mathrm{cN}$ for the maximum allowable tension.

\section{CONCLUSION}

We have shown that a combination of theoretical modelling and empirically determined relation between the yarn tension and the angular velocity of the yarn.

We have reached the following conclusions:

- In cross-wound packages, when the yarn unwinds backwards the angular velocity is higher as when the yarn unwinds forwards, which leads to oscillations in the yarn tension. Abrupt changes of tension occur at the edges of the package.

- Unwinding at high velocity $(2000 \mathrm{~m} / \mathrm{min})$ from regular cross-wound packages is impossible for package radii below $150 \mathrm{~mm}$.

\section{REFERENCES}

Padfield, D. G. (1958). The Motion and Tension of an Unwinding Thread. Proc. R. Soc., vol. A245, 382-407.

Barr, A. E. D., Catling, H. (1976). Manual of Cotton Spimmimg, Volume Five. Butterworth

Kothari,V.K.,Leaf G.A.V. (1979). The unwinding of yarns from packages, PartII: The theory of yarnunwinding.J.Text.Inst 70 (3)95-105

Fraser, W. B., Ghosh, T. K., Batra, S. K. (1992). On unwinding yarn from cylindrical package. Proc. R. Soc. Lond. A, 436 479-498

Praček, S. (2002). Modification of yarn unwinding dynamics. Dissertation, University of Ljubljana 